Looking out for danger: An attentional bias towards spatially predictable threatening stimuli.

Lies Notebaert*, Geert Crombez, and Stefaan Van Damme, Jan De Houwer Ghent University, Belgium

Jan Theeuwes

Vrije Universiteit, Amsterdam, the Netherlands

* Corresponding author: Lies Notebaert, Department of ExperimentalClinical and Health Psychology, Ghent University, Henri Dunantlaan 2, B9000 Ghent, Belgium. Tel: +32 926486 18,

Fax: +32 926464 89, e-mail: lies.notebaert@ugent.be 


\begin{abstract}
Attentional bias to threat is well established, however, the influence of spatial predictability on this attentional bias has never been investigated. Here we investigated how threat affects attentional capture and disengagement when its spatial location is predictable. Using a visual search paradigm, participants were required to identify a target inside one of a variable number of colored circles. One color (Conditioned Stimulus, CS+) was fear-conditioned using an electrocutaneous stimulus at tolerance level. In the experimental group the CS+ was made spatially predictable (occurred more often at one location in the visual display), while this was not the case in the control group. Results showed no complete automatic capture of attention by the CS+, but the experimental group did show more prioritization of the CS+ and less difficulty to disengage from the CS+ than the control group. Of further importance was the finding that the experimental group also attended to the location that was predictive of the CS+, even when no CS+ was presented. Findings are discussed in terms the effects of predictability on anxiety.
\end{abstract}

Keywords: attentional bias, threat, classical conditioning, predictability, hypervigilance, anxiety, fear 


\section{Looking out for danger: An attentional bias towards spatially predictable threatening stimuli.}

Imagine a colleague with a severe spider phobia. She never goes to sleep without first making sure the entire bedroom is spider-free, and she never goes to the basement of her house because she is petrified to run into a spider. Now, you both enter a meeting room for the weekly staff meeting. A spider is sitting on the wall just below the ceiling. Do you think this spider will capture your colleague's attention before yours?

Having an attentional bias for threatening stimuli is often considered to be adaptive (e.g., Öhman \& Mineka, 2001). It allows humans to quickly detect and identify potential threats, enabling an appropriate response. Mogg, Bradley, Miles, and Dixon (2004) proposed that attention is initially oriented to the threatening stimulus, after which it is strategically directed away from this stimulus. This idea that threatening stimuli automatically and unintentionally capture attention (see also Öhman \& Mineka, 2001) is in line with the phenomenological experience of attentional bias in patients suffering from phobia and anxiety (Ouimet, Gawronski, \& Dozois, 2009). Another line of research suggests that an important component of attentional bias to threat is a difficulty to direct attention away from threat once it is detected (see Weierich, Treat \& Hollingworth, 2008; Yiend, 2010). There has been a lot of empirical support for this disengagement hypotheses (Fox, Russo, Bowles \& Dutton, 2001; Cisler \& Koster, 2010), especially in (sub-) clinical populations (eg. Koster, Crombez, Verschuere, Van Damme \& Wiersema, 2006). 
Although this difficulty to disengage from threat is well established, there is no convincing empirical evidence that threatening information captures attention in a bottom-up way (Frischen, Eastwood, \& Smilek, 2008), meaning that the capturing stimulus receives attentional priority irrespective of other information in the environment (Theeuwes, Atchley \& Kramer, 2000). Appropriate paradigms to study attentional capture are those in which the number of competing stimuli is varied (Frischen et al., 2008), such as the visual search paradigm. A stimulus is said to capture attention when the time needed to identify the target is unaffected by the number of simultaneously presented distractors. That is, when the search slope is flat (Wolfe, 1998).

Recent studies investigating attentional capture by threat have revealed substantially flatter search slopes for threatening stimuli than for neutral stimuli, but not flat search slopes (e.g. Gerritsen, Frischen, Blake, Smilek, \& Eastwood, 2008; Notebaert, Crombez, Van Damme, De Houwer, \& Theeuwes, in press), indicating that threatening stimuli are prioritized over neutral stimuli, but do not automatically capture attention (Frischen et al., 2008). This prioritization of threat has also been found in other studies that have controlled for methodological confounds (Gerritsen et al., 2008; Horstmann, 2009).

In the search for experimental rigor and internal validity some variables concerning attentional bias to threat may have been overlooked or unduly eliminated. Spatial predictability might be one of these. Indeed, threatening events often do not occur at random locations. Patients often expect a confrontation with their feared object at particular locations (the basement for spider phobics, or the wings of an airplane for people with fear of flying,...). 
Patients probably scan locations were they expect threat much more often than those locations that are unlikely to contain threatening stimuli (Eysenck, 1992). It is likely then that spatial predictability contributes to the attentional bias to threat. As yet, the influence of spatial predictability on attentional bias not been investigated.

This study was designed to investigate the role of spatial predictability in attentional bias to threat using an adaptation of the visual search paradigm (Notebaert et al., in press). First, a threatening stimulus was created using a fear conditioning procedure, in which a previously neutral stimulus becomes a signal (Conditioned Stimulus, CS+) for an aversive, electrocutaneous stimulus (Van Damme, Crombez, Hermans, Koster, \& Eccleston, 2006). This permits control over the perceptual characteristics of the threatening stimulus. Second, we dissociated the threat value of a stimulus from its task relevance and made the CS+ not predictive of the target. This way, results cannot be easily explained by an instrumental task-strategy to search for the threatening stimulus. By making the threatening stimulus task-irrelevant, we also increased the external validity of our paradigm. Indeed, patients' attention is often captured by threatening information while they are engaged in another task (Crombez, Van Damme \& Eccleston, 2005). Third, the present design allows investigating to what extent threatening information is prioritized over neutral information (on trials in which the target is spatially congruent with the CS+) and the extent to which people have a difficulty to disengage from threat (on trials in which the target is spatially incongruent with the CS+). 
Participants were randomly assigned to two groups. In the experimental group, the threatening stimulus (CS+) was spatially predictable, meaning that on $80 \%$ of the trials it was presented at the same location in the display. In the control group, the CS+ was presented equally often at all locations. In this study, we were particularly interested in how a spatially predictable threatening stimulus affects attentional prioritization and disengagement from threat.

\section{Method}

\section{Participants}

Forty-seven students at Ghent University participated in exchange for course credit. The final sample of consisted of 23 participants in the experimental group ( 4 men, mean age $=18.1$ years, $S D=0.6$ ) and 21 in the comparison group ( 3 men, mean age $=18.6$ years, $S D=1.3$ ). All had normal or corrected-to-normal vision, and reported not to be color-blind. All participants gave their informed consent and were free to terminate the experiment at any time. No one made use of this option.

\section{Stimulus Material and Apparatus}

Participants were seated approximately $60 \mathrm{~cm}$ from the screen. A graphical representation of the stimuli can be seen in Figure 1. On each search trial, the computer display consisted of four or eight circles $\left(2.9^{\circ}\right.$ diameter) with a colored band $\left(0.5^{\circ}\right.$ and black outlined $)$ against a silver background color. These colored circles were spaced equally distant from the midpoint of an 
imaginary circle (radius of $6^{\circ}$ visual angle, center of the screen). All circles in the display had different colors (pink, blue, turquoise, yellow, green, orange, purple, red or grey, matched for intensity and luminance). The number of stimuli presented (four or eight) is the set size. The colored circles in the display contained a black line segment (extending $1^{\circ}$ ) in their centre. All line segments, except one, were tilted $\left(22.5^{\circ}\right.$ to either side of the horizontal or vertical plane). The other line segment was the target stimulus, and was either a horizontal or a vertical line segment.

\footnotetext{
${ }^{\star * *}$ Insert Figure 1 about here ${ }^{* * *}$
}

Electrocutaneous stimuli (US's) were delivered by a constant current stimulator (DIGITIMER, model DS7A), and administered to the inside of the wrist of the non-dominant forearm by two lubricated Fukuda standard $\mathrm{Ag} / \mathrm{AgCl}$ electrodes ( $1 \mathrm{~cm}$ diameter). The electrocutaneous stimuli consisted of a series of 38 rectangular pulses ( $2 \mathrm{~ms}$ in duration with an inter pulse interval of $6 \mathrm{~ms}$ ), and had a total duration of $300 \mathrm{~ms}$. The intensity of the US was the maximum intensity that participants were willing to tolerate.

\section{Procedure}

Upon arrival, trait anxiety was assessed with the Dutch trait version of the State Trait Anxiety Inventory (STAI-Trait; Spielberger et al., 1983). Next, the tolerance level of the electrocutaneous stimulus was individually determined. To increase the threat value of the electrocutaneous stimuli, participants were incorrectly led to believe that at some point during the experiment, the intensity of the US would exceed their tolerance level. 
During the practice phase, participants practiced the task with 23 trials (ten trials of set size 4 and 8 each, and three digit trials). No electrocutaneous stimuli were presented. A trial started with a fixation cross in the middle of the screen (500 ms duration). Thereafter, the search display was presented until a response was made. There was an inter trial interval of $250 \mathrm{~ms}$, and error feedback was given. Participants were instructed to focus on the fixation cross at the beginning of each trial, and to identify whether the target line was horizontal or vertical by responding as quickly as possible using a two button response box. Responses had to be made with the index and middle finger of the dominant hand. To ensure participants remained focused on the middle of the screen, we included digit trials where a digit (1 to 9) was presented at fixation for $100 \mathrm{~ms}$. Participants had to type in the digit, after which an inter trial interval of 500 ms followed.

During the acquisition phase, one color (Conditioned Stimulus, CS+) was made threatening by making it predictive of an electrocutaneous stimulus (Unconditioned Stimulus, US). All nine colors were used as CS+, counterbalanced across participants. Participants were instructed to find out which color was predictive of the electrocutaneous stimulus. In this phase, only colored circles without line segments were presented. To facilitate acquisition, this phase started with ten trials in which only one color was presented at fixation, successively followed by six trials of setsize 4 , and six trials of setsize 8. On half of the trials, the CS+ was presented. Six of the CS+ trials were followed by the US (50\% partial reinforcement schedule). The CS+ was presented for $750 \mathrm{~ms}$ and the US was delivered $300 \mathrm{~ms}$ after CS+ onset. At the 
end of the acquisition phase, participants had to report which color was predictive of the US.

During the experiment phase, there were two blocks, each consisting of 225 search trials and 15 digit trials (randomly intermixed). On search trials, half of the trials contained a horizontal line segment, the other half a vertical line segment. For each set size, the target was presented equally often at all possible locations. For each block, there were 75 trials of setsize 4, and 150 trials of setsize 8. In order for the threat-related color (CS+) to remain threatening and predictive of the US, we chose the following procedural aspects. First, only two thirds of the search trials contained CS+ in order to avoid habituation of the US and extinction of fear. Second, during each block, 10 trials in which the CS+ was followed by the US were added to avoid extinction.

There were three types of search trials. (1) Congruent trials, in which the target was presented in the CS+; (2) Incongruent trials, in which the CS+ was present but the target was depicted in another colored circle; (3) Baseline trials, in which a target, but no CS+ was present. Per setsize, one third of the trials were baseline trials. We opted for a ratio of congruent / incongruent trials based on the $1 / n$ procedure (where $n$ is set size) which prevents participants to strategically use the CS+ to localize the target because it ensures that the CS+ is not predictive of the target (Jonides \& Yantis, 1988). Thus, on the remaining trials, one in four trials with set size 4 and one in eight trials with set size 8 was a congruent trial. The residual trials were incongruent trials. For a schematic overview of these different trial types, see Figure 1. 
Participants were randomly (by lottery) assigned to one of two groups. In the control group, the CS+ was presented ad random equally often at all possible locations. In the experimental group we made the location of the CS+ predictable by presenting the CS+ at the same location (the "threat location") on $80 \%$ of the trials on which is was presented. This location was either at the top, bottom, most left or most right part of the display (location counterbalanced over participants). Thus, the CS+ was presented on the threat location at $80 \%$ of the congruent and $80 \%$ of the incongruent trials. On the remaining $20 \%$ of trials, the CS+ was presented randomly at all other possible locations.

At the end of the experiment, Likert Scales were used to assess to what extent the US was expected after presentation of the CS $+($ anchored by $0=$ Never and $9=$ Always), and the experienced fear during the presentation of the $\mathrm{CS}+($ anchored by $0=$ Not at all and $9=$ Very much $)$.

\section{Results}

\section{Data Trimming}

After the acquisition phase, one participant was not able to correctly identify the color that was predictive of the US. One participant had a mean accuracy on search trials below the sample's mean minus two and a half standard deviations, and one had a digit trial accuracy below the sample's mean minus two and a half standard deviations. The data of these three participants were removed from further analyses. In addition, search trials on which an US was presented were not taken into account for analyses because it 
is likely that the administration of the US interfered with reaction times on these trials. Also trials with response errors $(4.0 \%)$ and with outliers $(1.3 \%$, defined as reactions times that deviated more than two and a half standard deviations from the individual mean of correct responses, calculated for every trial type and setsize separately) were removed. For ease of comparison with the norms of Cohen (1988), we calculated effect sizes for dependent samples using the formula of Morris and DeShon (2002). An effect size of 0.20 is considered a small effect, around 0.50 a medium effect and 0.80 a large effect. Standard deviations of the means are reported between brackets.

\section{Self report data}

Trait anxiety scores did not differ between the experimental $(M=37.5$ [7.8]) and comparison group $(\mathrm{M}=39.0[10.5], \mathrm{t}<1)$. Both groups did not differ in their expectancy of the US after presentation of the CS+ $(M=4.0[2.7]$ for the experimental group, $M=4.2$ [2.7] for the control group; $t<1$ ) and were equally afraid when the $C S+$ was presented $(M=3.0[2.8]$ for the experimental group, $M$ $=2.4[2.3]$ for the control group; $t<1)$.

\section{Reaction time data}

We compared the three attentional bias indices between the two groups. For the facilitation effect, we compared individual mean reaction times on congruent trials to those on baseline trials. For the interference effect, we compared individual mean reaction times on incongruent to those on baseline trials. For the slope attenuation effect, we compared the search slope on congruent trials to the slope on baseline trials. For every subject, a search slope 
for each trial type was calculated by subtracting reaction times on set size 4 from those on set size 8 , and dividing it by 4 . The resulting number is the mean increase in reaction time per additional item in the display. A slope of $10 \mathrm{~ms}$ or less would indicate attentional capture (Wolfe, 1998). A graphical representation of the three attentional bias effects as a function of group can be found in Figure 2.

Because we wanted a pure measure of the effect of spatial predictability, we did not include all trials of the experimental group in our analyses. More specifically, for congruent trials, we selected only those $80 \%$ trials where the $\mathrm{CS}+$ (and hence also the target) was actually presented on the threat location. The other $20 \%$ of trials were removed from the analyses because this would introduce noise. Similarly, for the incongruent trials, we only selected the $80 \%$ trials where the $\mathrm{CS}+$ was presented at the threat location ${ }^{1}$. This was done because we are mainly interested in the effects of a spatially predictable threatening stimulus, when it is presented at that particular location (the "threat location"). We also subdivided the baseline trials of the experimental group. "Baseline trials at threat location" are those trials where the target line segment was presented at the threat location (one fourth of all baseline trials on set size 4 and one eighth of all baseline trials on set size 8). The "standard baseline trials" in the experimental group are baseline trials where the target line segment was presented at any of the other locations. Because we expected an allocation of attention to the threat location, even when no CS+ was present, we

\footnotetext{
${ }^{1}$ All results were similar when we included all $100 \%$ of the congruent and incongruent trials.
} 
only included the standard baseline trials in our analyses for the attentional bias indices. There was no difference between reaction times on the baseline trials of the control group and the standard baseline trials of the experimental group ( $t$ $<1)$.

First, we tested whether the control and experimental group both showed facilitation by threat using a 2 (Trial type: Congruent and Baseline) by 2 (Group: Experimental and Control) Repeated Measures ANOVA. Results showed a significant effect of Trial type $(F(1,42)=76.60, p<.001)$, no effect of Group $(F<$ 1) and no interaction $(F<1)$. Reaction times on congruent trials $(M=1262$ [255] ms) were shorter than reaction times on baseline trials ( $M=1508$ [263] ms) in both groups $\left(M_{\text {diff }}=272\right.$ [206] $\mathrm{ms}, d=1.04$ in the experimental group and $M_{\text {diff }}=$ $219[161] \mathrm{ms}, d=0.84$ in the control group).

Second, we investigated the interference effect by threat using a 2 (Trial type: Incongruent and Baseline) by 2 (Group) Repeated Measures ANOVA. Results showed a significant effect of Trial type $(F(1,42)=7.31, p=.01)$, indicating that reaction times on incongruent trials $(M=1547$ [285] ms) were longer than those on baseline trials. This effect was moderated by Group $(F(1,42)=4.91, p<.05)$, revealing that only the control group showed a significant difference between incongruent and baseline trials $\left(M_{\text {diff }}=72\right.$ [95] ms; $t(20)=3.45, p<.005, d=0.23)$. This interference effect was not significant in the experimental group $\left(M_{\text {diff }}=9\right.$ [101] $\left.\mathrm{ms}, t<1, d=0.03\right)$. The main effect of Group was not significant $(F<1)$. 
Third, to test whether the CS+ is captured or prioritized in the search task, we performed a 2 (Trial type: Congruent and Baseline) by 2 (Set size: 4 and 8 ) by 2 (Group) Repeated Measures ANOVA. Results showed significant main effects of Trial type $(F(1,42)=16.16, p<.001)$ and Set size $(F(1,42)=$ $381,19, p<.001)$, but no main effect of Group $(F(1,42)=1.96, p>.1)$. More importantly, there was a marginally significant three-way interaction $(F(1,42)=$ $4.00, p=.052)$. To disentangle this interaction, we compared the search slope on congruent and baseline trials in the two groups. In the control group, there is no significant flatter slope on congruent ( $M=161$ [84] ms) than on baseline trials $\left(\mathrm{M}=155\right.$ [37] $\mathrm{ms} ; M_{\text {diff }}=-6$ [87] $\left.\mathrm{ms} ; t<1, d=0.09\right)$, but the experimental group does show a significantly flatter slope on congruent (134 [59] ms) than on baseline trials $\left(M=170\right.$ [52] ms; $M_{\text {diff }}=36$ [61] ms; $t(22)=3.09, p<.01, d=$ $0.60)$.

*** Insert Figure 2 about here ***

Next, we investigated whether participants in the experimental group preferentially allocated their attention to the threat location. We compared the reaction times on standard baseline trials with the reaction times on the baseline trials at threat location. Reaction times on the baseline trials at threat location ( $M=1247$ [292] ms) were significantly shorter than on standard baseline trials $(M=1487$ [260] ms; $t(22)=5.51, p<.001, d=0.86)$. In addition, the slope for the baseline trials at threat location was significantly attenuated $(M$ $=118[83] \mathrm{ms})$ in comparison with the slope of the standard baseline trials $(t(22)$ $=3.41, p<.005, d=0.73)$. This result reveals that the experimental group 
prioritized attention towards the threat location even when no CS+ was presented.

To examine whether any of the attention effects (facilitation, interference and slope attenuation) are influenced by anxiety, we calculated correlations between trait anxiety scores and all attention indexes. In the control group, we found a significant correlation between the amount of interference caused by the CS+ and trait anxiety scores $(r(21)=.44, p<.05)$. In the experimental group none of the correlations reached significance (all $p>.1$ ).

\section{Discussion}

This study investigated the effect of spatial predictability of the feared object (the $\mathrm{CS}_{+}$) on attentional capture by threat. The results can be readily summarized. First, in the control group where the CS+ was not spatially predictable, the CS+ caused both facilitation and interference in the visual search task. However, the lack of slope attenuation on congruent trials compared to baseline trials indicates that the CS+ was not prioritized over the other stimuli. Second, when the CS+ was spatially predictable, the CS+ was prioritized over other stimuli, but did not automatically capture attention.

Although a strong attentional bias is present, our data do not reveal that the CS+ automatically captures attention, even when it was spatially predictable. Although there are theoretical (Mogg et al., 2004; Öhman et al., 2001) and clinical (Ouimet et al., 2009) reasons to expect attentional capture by threat, our results did not show this effect. However, we did found that the CS+ 
was prioritized when it was spatially predictable. The slope on congruent trials was flatter when the CS+ was predictable than when it was not predictable. There are several explanations for this prioritization effect. First, it is possible that unattended threatening stimuli guide attention by reducing the number of saccades required to locate threat (see Reynolds et al, 2008). A second possibility is that, when a threatening stimulus is present, people are faster in disengaging attention from neutral distractors (for a related but distinct phenomenon, see Becker, 2009). In contrast to this automatic capture hypothesis, our data suggest that the enhanced prioritization of threat is the result of strategic, top-down processes. Indeed, we found an equally large slope attenuation effect for the baseline trials at the threat location. A plausible interpretation is that participants strategically scan the threat location for the presence of the CS+. This idea is in line with a eye movement studies in spider phobics. Lange and colleagues (2004) reported that while watching television, participants with spider phobia paid more attention to a spider present in the room (as deduced from longer viewing times) and showed more scanning of the environment than non-phobic participants. Note however, that in our paradigm a scanning strategy is not instrumental for the task at hand: the threat location was not more predictive of the target than any other location.

Of further interest was that we observed an interference effect in the control group but not in the experimental group. The interference effect in the control group can be explained by a delayed disengagement from the threatening stimulus. This phenomenon has been observed repeatedly in previous research (Cisler, Bacon \& Williams, 2009). The finding that the 
magnitude of the interference effect in the control group correlated with participants' anxiety scores renders support for this explanation. The lack of an interference effect in the experimental group might be due to the fact that the spatial predictability of the CS+ prepared participants for a quicker disengagement from the threatening stimulus. This might be because predictive events seem to be less distressing and disruptive than unpredictive events (Crombez, Baeyens, \& Eelen, 1994; Mineka \& Kihlstrom, 1978; Fonteyne, Vervliet, Hermans, Baeyens \& Vansteenwegen, 2009). There is already evidence showing that temporal unpredictability of aversive events is an important factor in the etiology and maintenance of anxiety disorders (Lohr, Olatunji \& Sawchuk, 2007). It has been shown that temporally predicted panic attacks lead to a decrease in anxiety and worry levels, whereas unpredicted panic attacks increases worry and anxiety (Craske, Glover \& Decola, 1995). Some behavioural therapies for anxiety disorders also aim at increasing the predictability of anxiety symptoms (Arch \& Craske, 2008). However, the role of spatial (un)predictability is largely unexplored. Our study suggests that it is worthwhile to also focus on the spatial predictability. If patients can anticipate the location of a threatening experience, it seems to be easier to subsequently disengage attention from it and this might (in line with research on temporal predictability) diminish anxiety. On the other hand, spatially predictable threatening stimuli were also prioritized more, and it is known that hypervigilance plays an important role in the origin and maintenance of anxiety disorders (Eysenck, 1992). Therefore, more research is needed to corroborate the role of spatial predictability on attentional processing and anxiety, especially 
in clinical populations. This way, it can be investigated whether disengagement and prioritization are differentially affected in anxiety patients, which would shed more light on the components that are crucial to anxiety disorders.

There are some limitations to this study. First, one should be careful in generalizing the results in healthy students to clinical samples. Second, although participants did not benefit from allocating their attention to the threat location, there were also no negative consequences for doing this. It is worthwhile to investigate whether the pattern of results changes when attention to a spatially predictable threatening stimulus is detrimental for the task at hand. 


\section{References}

Arch, J.J., \& Craske, M.G. (2008). Acceptance and Commitment Therapy and Cognitive Behavioral Therapy for Anxiety Disorders: Different Treatments, Similar Mechanisms? Clinical Psychology-Science and Practice, 15, 263-279.

Becker, M.W. (2009). Panic Search: Fear Produces Efficient Visual Search for Nonthreatening Objects. Psychological Science, 20, 435-437.

Cisler, J.M., Bacon, A.K., \& Williams, N.L. (2009). Phenomenological Characteristics of Attentional Biases Towards Threat: A Critical Review. Cognitive Therapy and Research, 33, 221-234.

Cisler, J.M., \& Koster, E.H.W. (2010). Mechanisms of attentional biases towards threat in anxiety disorders: An integrative review. Clinical Psychology Review, 30, 203-216.

Cohen, J. (1988). Statistical power analysis for the behavioral sciences (2nd ed.). Hillsdale, NJ: Erlbaum.

Craske, M. G., Glover, D., \& Decola, J. (1995). Predicted Versus Unpredicted Panic Attacks - Acute Versus General Distress. Journal of Abnormal Psychology, 104, 214-223.

Crombez, G., Baeyens, F., \& Eelen, P. (1994). Sensory and temporal information about impending pain: The influence of predictability on pain. Behaviour Research and Therapy, 32, 611-622. 
Crombez, G., Van Damme, S., \& Eccleston, C. (2005). Hypervigilance to pain: An experimental and clinical analysis. Pain, 116, 4-7.

Eysenck, M.W. (1992). Anxiety: The cognitive perspective. Erlbaum, Hillsdale, NJ.

Fonteyne, R., Vervliet, B., Hermans, D., Baeyens, F., \& Vansteenwegen, D. (2009). Reducing chronic anxiety by making the threatening event predictable: An experimental approach. Behaviour Research and Therapy, 47, 830-839.

Fox, E., Russo, R., Bowles, R., \& Dutton, K. (2001). Do threatening stimuli draw or hold visual attention in subclinical anxiety? Journal of Experimental Psychology-General, 130, 681-700.

Frischen, A., Eastwood, J. D., \& Smilek, D. (2008). Visual search for faces with emotional expressions. Psychological Bulletin, 134, 662-676.

Gerritsen, C., Frischen, A., Blake, A., Smilek, D., \& Eastwood, J. D. (2008). Visual search is not blind to emotion. Perception \& Psychophysics, 70, 1047-1059.

Horstmann, G. (2009). Visual search for schematic affective faces: Stability and variability of search slopes with different instances. Cognition \& Emotion, 23, 355-379.

Jonides, J., \& Yantis, S. (1988). Uniqueness of abrupt visual onset in capturing attention. Perception \& Psychophysics, 43, 346-354. 
Koster, E.H.W., Crombez, G., Verschuere, B., Van Damme, S., \& Wiersema, J. R. (2006). Components of attentional bias to threat in high trait anxiety: Facilitated engagement, impaired disengagement, and attentional avoidance. Behaviour Research and Therapy, 44, 1757-1771.

Lange, W.G.T., Tierney, K.J., Reinhardt-Rutland, A.H., \& Vivekananda-Schmidt, P. (2004). Viewing behaviour of spider phobics and non-phobics in the presence of threat and safety stimuli. British Journal of Clinical Psychology, 43, 235-243.

Lohr, J.M., Olatunji, B.O., \& Sawchuk, C.N. (2007). A functional analysis of danger and safety signals in anxiety disorders. Clinical Psychology Review, 27, 114-126.

Mineka, S., \& Kihlstrom, J.F. (1978). Unpredictable and Uncontrollable Events New Perspective on Experimental Neurosis. Journal of Abnormal Psychology, 87, 256-271.

Mogg, K., Bradley, B.P., Miles, F., \& Dixon, R. (2004). Time course of attentional bias for threat scenes: Testing the vigilance-avoidance hypothesis. Cognition \& Emotion, 18, 689-700.

Morris, S.B., \& DeShon, R.P. (2002). Combining effect size estimates in metaanalysis with repeated measures and independent-groups designs. Psychological Methods, 7, 105-125. 
Notebaert, L., Crombez, G., Van Damme, S., De Houwer, J., \& Theeuwes, J. (in press). Signals of threat do not capture, but prioritize attention: a classical conditioning approach. Emotion

Öhman, A., \& Mineka, S. (2001). Fears, phobias, and preparedness: Toward an evolved module of fear and fear learning. Psychological Review, 108, 483-522.

Ouimet, A.J., Gawronski, B., \& Dozois, D.J.A. (2009). Cognitive vulnerability to anxiety: A review and an integrative model. Clinical Psychology Review, 29, 459-470.

Spielberger, C.D., Gorsuch, R.L., Lushene, R., Vagg, P.R., \& Jacobs, G.A. (1983). Manual for the State-Trait Anxiety Inventory. Palo Alto, CA: Consulting Psychologists Press.

Theeuwes, J., Atchley, P., \& Kramer, A.F.(2000). On the time course of topdown and bottom-up control of visual attention. In S., Monsell \& J., Driver (Eds.). Attention \& Performance ((p. 105-125). Cambridge: MIT Press.

Van Damme, S., Crombez, G., Hermans, D., Koster, E.H.W., \& Eccleston, C. (2006). The role of extinction and reinstatement in attentional bias to threat: A conditioning approach. Behaviour Research and Therapy, 44, 1555-1563.

Wolfe J. (1998). Visual search. In Pashler H. (ed.). Attention. University College London Press, London, UK. 


\section{Acknowledgements}

This research was funded by grants B/07386/02 and BOF/GOA2006/001 of Ghent University. 


\section{Figure Captions}

Figure 1. Schematic examples of the different stimulus displays (not to scale). Spotted circles represent the CS+. Panel A: Congruent trial of set size 4 with horizontal target presented at the same location as the CS+. Panel B:

Incongruent trial of set size 8 with vertical target presented at a different location than the CS+. Panel C: Baseline trial of set size 8 with vertical target and no CS+ present.

Figure 2. Mean facilitation effect (baseline minus congruent trials), interference effect (incongruent minus baseline trials), and slope attenuation effect (slope baseline minus slope congruent trials) for the control group and experimental group (standard baseline trials). Error bars represent standard errors of the mean. 